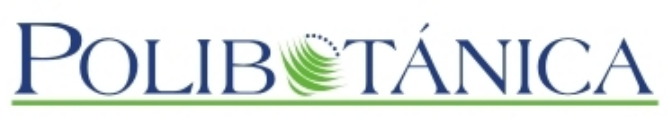

\title{
Polibotánica
}

ISSN electrónico: 2395-9525

polibotanica@gmail.com

Instituto Politécnico Nacional

México

http://www.polibotanica.mx

\section{LA FAMILIA ROSACEAE EN MÉXICO}

\section{THE FAMILY ROSACEAE IN MEXICO}

\section{Rzedowski, J.}

LA FAMILIA ROSACEAE EN MÉXICO.

THE FAMILY ROSACEAE IN MEXICO.

\section{POLIBETÁNICA}

Instituto Politécnico Nacional
Núm. 51: 1-16 México. Enero 2021

DOI: $10.18387 /$ polibotanica.51.1 
LA FAMILIA ROSACEAE EN MÉXICO

THE ROSACEAE FAMILY IN MEXICO

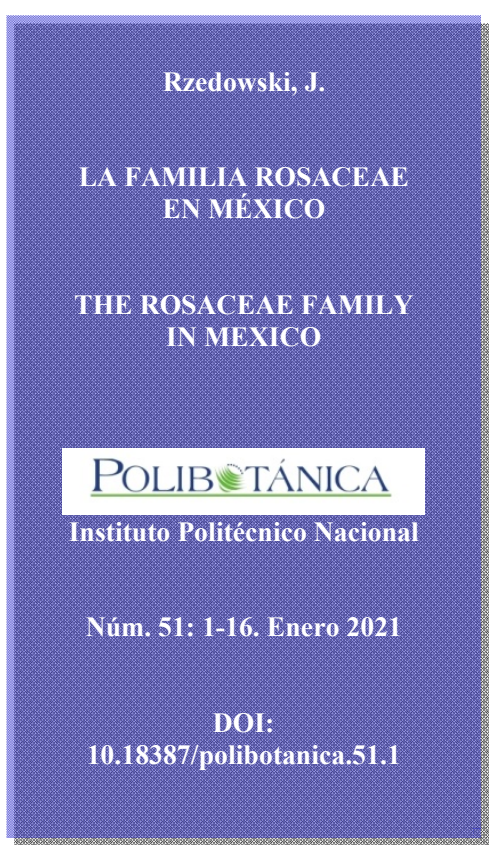

J. Rzedowski / jerzedowski@gmail.com Instituto de Ecología, A.C. Centro Regional del Bajio Apartado postal 382 61600 Pátzcuaro, Michoacán

RESUMEN: Se presenta un inventario actualizado de los componentes silvestres de la familia Rosaceae en México. Se enumeran 192 especies (pertenecientes a 34 géneros), de las cuales 11 no son nativas y 26 se conocen de un solo estado; no se encontraron ejemplares ni citas de su existencia en Campeche, Quintana Roo, Tabasco y Yucatán. Se realiza un somero análisis de la ubicación geográfica y ecológica de sus componentes, enfatizando su significativa participación en los matorrales xerófilos en el territorio de la República.

Palabras clave: inventario, matorral xerófilo, México, Rosaceae.

ABSTRACT. An updated inventory of spontaneous members of the Rosaceae family in Mexico is presented. 192 species (belonging to 34 genera) are listed; 11 of them are not native and 26 are only reported from one of the states. No references were found of their occurrence in the states of Campeche, Quintana Roo, Tabasco and Yucatan. A brief survey of the geographic and ecological location of the elements of the family is performed, emphasizing their significant participation in the composition of the xerophilous flora in Mexico.

Key words: inventory, Mexico, Rosaceae, xerophilous scrub.

\section{INTRODUCCIÓN}

Las Rosaceae constituyen uno de los grandes grupos de las angiospermas, pues incluyen alrededor de 90 géneros y probablemente más de 3,000 especies. En su totalidad es un conjunto casi cosmopolita, aunque es particularmente diverso en áreas de clima templado, sobre todo, en el Hemisferio boreal. Su participación en la flora intertropical termófila es escasa y en muchos lugares nula.

Varios de sus componentes son de mayor importancia para la humanidad. Aquí pertenecen diversos árboles y también varias plantas herbáceas que son básicas en la fruticultura a nivel mundial, destacando la almendra (Prunus dulcis (Mill.) D. A. Webb, la cereza (Prunus avium (L.) L., P. cerasus L.), el chabacano (Prunus armeniaca L.), la ciruela (Prunus domestica L., P. salicina Lindl.), el durazno (Prunus persica (L.) Betsch), la frambuesa (Rubus idaeus L.), la fresa (Fragaria x ananassa (Duchasne ex Weston) Duchasne ex Rozier), la manzana (Malus domestica (Suckow) Barkh.), el membrillo (Cydista oblonga Mill.), el níspero (Eriobotrya japonica Thunb.), la pera (Purus communis L.) y la zarzamora (Rubus spp., mayormente híbridos). No pocos destacan por sus atractivas flores y se cultivan para tal propósito, ocupando la rosa (Rosa spp., mayormente híbridos) el lugar más prominente en este conjunto. 
En México, además, no dejan de ser relevantes dos frutales nativos, a mencionar el capulín (Prunus serotina ssp. capuli (Cav.) McVaugh) y el tejocote (Crataegus mexicana DC.), que han sido objeto de selección, cultivo y consumo desde tiempos prehispánicos.

En su actual circunscripción la familia se considera monofilética (Potter et al., 2002). Sin embargo, la historia de su taxonomía revela que ha pasado por diferentes contextos e interpretaciones. Así, por ejemplo, en el trabajo de principios del siglo pasado Rydberg (19081918), que consiste en la revisión de todas sus especies de América del norte (incluyendo México, Centroamérica y las Antillas), no se trataron los grupos de Maloideae y Prunoideae, que en esa época muchos autores reconocían como familias independientes.

Ese mismo criterio lo sostuvo Standley (1922c) quien, en su obra sobre árboles y arbustos de México, dividió tal conjunto de plantas en Rosaceae, Malaceae y Amygdalaceae. A su vez, en este último grupo estuvieron incluidos también varios géneros que en el presente se definen como componentes de Chrysobalanaceae.

Las mencionadas contribuciones de Rydberg y de Standley siguen funcionando como importantes fuentes de información e instrumentos de identificación de muchos componentes mexicanos de esta familia.

Entre los botánicos actuales que han abordado la sistemática de algunos de sus géneros mexicanos procede mencionar a Barbara Ertter, James S., Henrickson y a James B. Phipps.

En términos generales, las Rosaceae se han ganado el calificativo de taxonómicamente difíciles a raíz de fuertes controversias en la delimitación de sus taxones, sobre todo a nivel de especie en muchos de sus géneros. Así por ejemplo para Rubus se han descrito más de 2,200 especies, de las cuales varios autores no aceptan más de 300. De acuerdo con Turner (2011), al género mayormente mexicano Malacomeles le corresponden cinco especies, mientras que Campbell (2014) sólo reconoce la existencia de dos.

Tal circunstancia significa que a lo largo del tiempo a menudo se han modificado y se siguen modificando las circunscripciones de algunos géneros y de numerosas especies y en consecuencia en los herbarios una buena proporción de los ejemplares de esta familia no lleva el nombre que en la actualidad le toca, con todas sus respectivas secuelas.

De este modo no deben sorprender al lector varias de las discrepancias que encontrará entre la aproximación presentada en este trabajo y otras contribuciones al conocimiento de la familia en México, que por cierto son bastante escasas.

La dificultad en la identificación de muchos ejemplares de Rosaceae es también la principal causa de las relativamente pocas adiciones de sus novedades a la flora de México publicadas en los últimos 100 años.

El principal objetivo de la presente contribución consiste en la integración de un inventario actualizado de los componentes silvestres de la familia en México, así como de su ubicación geográfica y ecológica.

\section{MÉTODO}

El catálogo se integró mediante una extensa consulta de la literatura y de varios portales electrónicos. Para tal propósito resultaron ser substanciales las siguientes obras:

- $\quad$ Rosaceae, Malaceae y Amygdalaceae, in: Trees and shrubs of Mexico (Standley, 1922); 
- Rosaceae, in: North American Flora (Rydberg, 1908-1918);

- $\quad$ Rosaceae, in: Checklist of the native vascular plants of Mexico (Villaseñor, 2016);

- $\quad$ Rosaceae, in: Flora of North America, north of Mexico (varios autores, 2014);

- $\quad$ Rosaceae, in: Flora Mesoamericana (Barrie et al., 2015);

- Rosaceae, in: Annotated checklist of the vascular plants of Baja California, Mexico (Rebman et al., 2016);

- $\quad$ Rosaceae, in: A flora of the Chihuahuan desert region (Henrickson \& Johnston, 1997);

- $\quad$ Rosaceae, in Flora fanerogámica del Valle de México (Calderón de Rzedowski, 2001);

- Rosaceae, in Flora del Bajío y de regiones adyacentes (Rzedowski \& Calderón de Rzedowski, 2005);

- $\quad$ Rosaceae, in Flora del Valle de Tehuacán-Cuicatlán (Martínez-Ramírez, 2014).

Se revisaron también numerosos trabajos relacionados con la sistemática y filogenia de los géneros mexicanos de la familia, una parte de los cuales se encuentra en la literatura citada.

Se procuró localizar e incorporar a la lista los elementos nuevos de Rosaceae descritos del territorio de la República en los siglos XX y XXI.

Con el objeto de completar y actualizar la información relativa a la distribución geográfica conocida de las especies se consultaron principalmente los portales electrónicos 'Colecciones Biológicas UNAM', 'Tropicos' y 'GBIF'.

\section{RESUlTADOS}

En este censo se reconocen 34 géneros, de los cuales dos (Lindleya y Xerospiraea) restringen su distribución al territorio de la República y tres solamente incluyen especies introducidas y más o menos naturalizadas al menos en alguna parte del país. Los mejor representados son: Rubus (36 spp.), Potentilla (34 spp., de las cuales 21 son endémicas de México), Prunus (32 spp.), Crataegus (13 spp.) y Lachemilla (10 spp.).

A nivel de especie se enlistan 192 elementos, de los cuales 74 (38.5\%) únicamente se han registrado dentro de los límites del país y 11 con seguridad no son nativas. 26 se han colectado en un solo estado. No se han visto ejemplares ni citas de Rosaceae espontáneas de Campeche, Quintana Roo, Tabasco y Yucatán (véase Cuadro 1). 
Cuadro 1. Números de especies registradas y de las localmente endémicas en los estados de la República.

\begin{tabular}{|l|c|c|}
\hline \multicolumn{1}{|c|}{ Estado } & Número de especies & Número de especies endémicas estatales \\
\hline Chiapas & 52 & 0 \\
\hline Oaxaca & 51 & 3 \\
\hline Veracruz & 51 & 1 \\
\hline Hidalgo & 46 & 5 \\
\hline Chihuahua & 43 & 0 \\
\hline Puebla & 39 & 1 \\
\hline Estado de México & 38 & 3 \\
\hline Coahuila & 37 & 1 \\
\hline Baja California & 36 & 1 \\
\hline Michoacán & 34 & 3 \\
\hline Nuevo León & 34 & 2 \\
\hline Querétaro & 30 & 0 \\
\hline Ciudad de México & 28 & 1 \\
\hline Durango & 28 & 0 \\
\hline Jalisco & 28 & 0 \\
\hline San Luis Potosí & 27 & 0 \\
\hline Tamaulipas & 26 & 1 \\
\hline Guerrero & 22 & 1 \\
\hline Guanajuato & 21 & 0 \\
\hline Morelos & 19 & 0 \\
\hline Tlaxcala & 18 & 0 \\
\hline Sonora & 16 & 0 \\
\hline Zacatecas & 13 & 0 \\
\hline Aguascalientes & 10 & 1 \\
\hline Nayarit & 9 & 0 \\
\hline Sinaloa & 8 & 0 \\
\hline Colima & 5 & 0 \\
\hline Baja California Sur & 4 & 0 \\
\hline Campeche & 0 & 0 \\
\hline Quintana Roo & 0 & 0 \\
\hline Tabasco & 0 & 0 \\
\hline Yucatán & & 0 \\
\hline & 0 & 0 \\
\hline
\end{tabular}

\section{DISCUSIÓN}

Acaena es un género de afinidad austral-antártica, presente también a lo largo de los Andes. Aunque Lachemilla y varias especies de Rubus muestran afinidad geográfica andina, los dos géneros probablemente son de ascendencia boreal, al igual de las demás Rosaceae de México que señalan la relación con la flora holártica, en particular con la de Estados Unidos de América. Esta vinculación se pone de particular manifiesto en el sureste del país, donde en cuatro estados, caracterizados por su flora termófila, faltan por completo sus representantes. Son escasos también en Sinaloa y en Colima y tal carácter se expresa a todo lo largo de la tierra caliente en la vertiente pacífica, incluyendo las porciones media e inferior de la cuenca del Balsas.

Llama la atención la existencia de numerosos representantes de la familia en el sector noroccidental de Baja California, donde prevalece el clima con lluvias concentradas entre diciembre y abril. Esta porción de México forma parte de la provincia florística californiana, en la cual las Rosaceae son frecuentes y abundantes. 
A grandes rasgos cabe apreciar que en nuestro país los representantes de esta familia son elementos de un importante número de comunidades vegetales, aunque se registran relativamente pocos en los bosques tropicales. También son escasos los de la vegetación acuática y subacuática. Son bastantes los componentes de comunidades secundarias, pero no hay ninguno que se comporte de manera preferente como maleza ruderal o arvense. En su mayoría habitan los bosques de coníferas y de encino. Aunque hay entre ellos más de 20 especies francamente arbóreas (Crataegus, Photinia, Prunus), éstas no llegan a ser dominantes ni numéricamente importantes en la vegetación.

De singular interés resulta ser la significativa presencia cuantitativa de Rosaceae en los matorrales xerófilos de México. Son de esta afinidad ecológica: Chamaebatia australis, casi todas las especies de Cercocarpus, todas las de Adenostoma, de Purshia y de Vauquelinia, varias de Malacomeles, así como los géneros monotípicos Fallugia, Heteromeles, Lindleya y Xerospiraea, los dos últimos endémicos de nuestro país. Son arbustos o pequeños árboles, en su mayoría perennifolios, con especiales adaptaciones en sus hojas para resistir largas temporadas de sequía. Algunos aspectos del origen de varios componentes de este grupo han sido tratados en el reciente artículo de Vásquez-Cruz y Sosa (2020).

En este último contexto cabe señalar que Velasco de León \& Cevallos-Ferriz (2000) reportaron la presencia de hojas de Cercocarpus en el Oligoceno en el estado de Puebla.

En el norte de Baja California los arbustos del género Adenostoma son elementos dominantes o codominantes del 'chaparral', comunidad de amplia distribución en el área; Cercocarpus y Malacomeles también forman parte de este matorral. De la sierra de Zapalinamé, ubicada en la cercanía de la ciudad de Saltillo, en el sur de Coahuila, se ha descrito (Arce \& Marroquín, 1985; Encina Domínguez et al., 2012) la existencia del 'matorral de rosáceas', constituido principalmente por especies de Purshia, Malacomeles, Lindleya y Cercocarpus.

En la flora de zonas francamente áridas de Sudamérica, de Asia y de África es mucho menos frecuente y conspicua la presencia de representantes de esta familia.

En general, el conocimiento actual de los componentes mexicanos de Rosaceae dista mucho de ser satisfactorio. Con seguridad colectas intensas en regiones menos exploradas del país, como muchos rincones de la Sierra Madre Occidental y de la Sierra Madre del Sur, revelarán la existencia de un contingente adicional de sus especies. La taxonomía de los grandes géneros Potentilla, Rubus, al igual que de la mayor parte de los componentes de Prunus no ha sido tratada ni revisada por cerca o más de 100 años. Poco se sabe de su morfología y anatomía. Faltan estudios autoecológicos, de la ecología de sus poblaciones, del papel que desempeñan en los ecosistemas. A pesar del hecho de que muchos de sus elementos tienen flores vistosas, el autor no ha podido encontrar contribuciones al conocimiento de la biología de su polinización ni tampoco de la dispersión de sus frutos y/o semillas. Aunque se sabe que las especies de Cercocarpus, de Chamaebatia y de Purshia viven en simbiosis con bacterias fijadoras de nitrógeno del género Frankia, al parecer no ha habido intentos de conocer la significación de esta propiedad en la vegetación de México.

Las Rosaceae forman parte importante de la biota y de la cubierta vegetal de nuestro país y será de mucha trascendencia que los botánicos mexicanos dediquen esfuerzos para su mejor cognición, aprovechamiento y conservación.

\section{AGRADECIMIENTOS}

La doctora Rosaura Grether, los doctores Abisaí García Mendoza y James S. Henrickson, así como tres revisores anónimos, tuvieron la amabilidad de examinar y sugerir correcciones al manuscrito. Se les agradece profundamente esta gentileza. 


\section{CATÁlOGO DE LAS ESPECIES MEXICANAS DE ROSACEAE}

El catálogo está organizado en secuencia alfabética de géneros y especies. No se tomaron en cuenta las entidades infraespecíficas. Para cada género se enlistan los nombres de los componentes registrados, acompañados de la información de su distribución geográfica conocida en México, señalada por los estados de la República. El signo * corresponde a elementos de área totalmente restringida a México, el ** a los endémicos a un solo estado, $\mathrm{el}^{\circ} \mathrm{a}$ los que no son nativos.

Acaena Mutis ex L.

Género de unas 45 especies mayormente distribuidas en el Hemisferio Sur (Sudamérica, Australia, Nueva Zelanda). Una se registra de Hawai. Otra, de área disyunta, crece en California; la presente en México se extiende al norte desde Bolivia hasta el centro de nuestro país. Referencia: Bitter, 1910.

A. elongata L., CdMx, Chis, Col, Gro, Hgo, Jal, Méx, Mich, Mor, Oax, Pue, Qro, Tlax, Ver

Adenostoma Hook. \& Arn.

Género de dos especies distribuidas en el sur de California, así como en Baja California.

Referencia: Standley, 1922.

A. fasciculatum Hook. \& Arn., BC

A. sparsifolium Torr., BC

\section{Agrimonia L.}

Genéro de alrededor de 20 especies distribuidas en América, Eurasia y Sudáfrica.

Referencia: Kline \& Sorensen, 2008.

A. gryposepala Wallroth, Chih, Coah, NL

*A. pringlei Rydb., Hgo, Pue, Qro, SLP, Tamps. Ver

A. striata Michx., CdMx, Chih, Hgo, Sin, Son, Tamps.

Amelanchier Medic.

Género de unas 20 especies mayormente de Norteamérica, unas pocas en Eurasia y norte de África. Referencia: Spellenberg et al., 1995.

A. utahensis Koehne, BC, Chih

\section{Aphanes L.}

Género de unas 20 especies, distribuidas en Norte y Sudamérica, Eurasia y norte de África, introducido en Australia y Nueva Zelanda. Algunos autores prefieren ubicarlo como parte de Alchemilla L. Referencia: McNeill \& Ertter, 2014.

A. occidentalis (Nutt.) Rydb., BC

\section{Cercocarpus Kunth}

Género de componentes mayormente xerófilos, distribuido en el oeste de Estados Unidos de América y en México, de taxonomía extraordinariamente complicada y controvertida, pues mientras algunos autores reconocen 21 especies, otros las reducen a seis. En esta contribución se aceptan nueve. Referencias: Martin, 1950; Lis, 1992.

C. betuloides Nutt., BC

C. breviflorus A. Gray, Chih, Coah, Dgo, Hgo, NL, Qro, SLP, Son, Tamps, Zac

${ }^{*}$ C. fothergilloides Kunth, Coah, Hgo, NL, Oax, Pue, Qro, SLP, Tamps

C. ledifolius Nutt., BC

*C. macrophyllus C. Schneid., Ags, Chih, Coah, Col, Dgo, Gro, Gto, Hgo, Jal, Méx, Mich, NL, Oax, Pue, Qro, Sin, SLP, Tamps, Ver, Zac

${ }^{* *}$ C. mexicanus Henrickson, NL

C. montanus Raf., Coah

${ }^{*}$ C. pringlei (C. Schneid.) Rydb., Gro, Hgo, Oax, Pue, Qro

*C. rzedowskii Henrickson, NL, SLP, Tamps 
Chamaebatia Benth.

Género de dos especies nativas de California y Baja California. Referencia: Henrickson, 2014.

C. australis (Brandegee) Abrams, BC

${ }^{\circ}$ Cotoneaster Medic.

Género de unas 400 especies nativas de Eurasia y norte de África. Muchas se cultivan como plantas de ornato y no pocas se han naturalizado en otras partes del mundo.

${ }^{\circ}$ C. pannosus Franch., CdMx, Hgo, Mich

\section{Crataegus L.}

Género de unas 230 especies, distribuidas originalmente en regiones de clima templado del Hemisferio Norte. Actualmente no pocas han sido introducidas y naturalizadas en otras partes del mundo.

Los frutos de muchas son comestibles y varias se han sometido al cultivo y a la comercialización a escala por lo general limitada. Las especies mexicanas se conocen como tejocotes. Referencia: Phipps, 1997.

${ }^{*}$ C. aurescens J. B. Phipps, Coah, NL

${ }^{*}$ C. boroussana J. B. Phipps, Coah, NL

**C. cuprina J. B. Phipps, NL

*C. gracilior J. B. Phipps, Hgo, NL, Oax, Pue, Qro, SLP, Tamps, Ver

${ }^{*}$ C. grandifolia J. B. Phipps, Coah, NL

C. greggiana Eggl., Coah, Hgo, NL, Tamps

**C. johnstonii J. B. Phipps, Coah

C. lindenii Stapf, Chis

C. mexicana DC., CdMx, Chis, Gro, Gto, Hgo, Jal, Méx, Mich, Mor, Oax, Pue, Qro, SLP, Tlax, Ver, Zac

${ }^{*}$ C. rosei Eggl., Chih, Coah, Dgo, Gto, Hgo, NL, Sin, SLP, Tamps

${ }^{*}$ C. sulfurea J. B. Phipps, Coah, NL

C. tracyi Ashe ex Eggl., Coah, NL

C. uniflora Münchh., Tamps

Drymocallis Fourr. ex Rydb.

Género de unas 30 especies distribuidas en el Hemisferio Norte, en su mayoría en el oeste norteamericano. Solamente una en el NW de Baja California. Referencia: Ertter, 2007.

D. glandulosa (Lindl.) Rydb., BC

${ }^{\circ}$ Eriobotrya Lindl.

Género de unas 30 especies nativas de Asia. Una de ellas, el níspero, es ampliamente cultivada por sus frutos comestibles y localmente naturalizada en otras partes del mundo.

${ }^{\circ}$ E. japonica (Thunb.) Lindl., Oax

Fallugia Endl.

Género monotípico distribuido en zonas áridas del oeste de Estados Unidos de América y norte de México. Referencia: Henrickson, 2001.

F. paradoxa (D. Don) Endl. ex Torr., BC, Chih, Coah, Dgo, Son, Zac

\section{Fragaria L.}

Género de 24 especies, distribuidas en las regiones de clima templado del Hemisferio Norte, en los Andes sudamericanos, así como en Polinesia. Algunos autores prefieren ubicar a Fragaria en la sinonimia de Potentilla. Referencia: Staudt, 1999.

F. vesca L., Ags, BC, BCS, CdMx, Chih, Chis, Dgo, Gro, Gto, Hgo, Jal, Méx, Mich, Mor, NL, Oax, Pue, Qro, SLP, Son, Tamps, Tlax, Ver, Zac 
Geum L.

Género de unas 45 especies distribuidas en América, Eurasia, Sudáfrica, Australia y Nueva Zelanda. Referencia: Gajewski, 1956.

G. aleppicum Jacq., CdMx, Chih, Coah, Hgo, NL, Pue

G. canadense Jacq., CdMx, Chis

*G. mexicanum Rydb., Hgo, Ver

Heteromeles M. Roem.

Género de una especie nativa de California y Baja California. Referencia: Phipps, 1992.

H. arbutifolia (Lindl.) M. Roem., BC

Holodiscus (K. Koch) Maxim.

Género esencialmente mexicano, con elementos de circunscripción muy controvertida, globalmente distribuido de Canadá a Venezuela, con cinco especies actualmente reconocidas. Lis (1990) propone someter a H. pachydiscus a la sinonimia de H. discolor. Referencias: Ley, 1943; Lis, 1990.

H. argenteus (L.f.) Maxim., Chis, Oax

H. discolor (Pursh) Maxim., BC, Chih, Dgo, NL, SLP, Son, Zac

H. fissus (Lindl.) C. K. Schneid., Chis, Dgo, Gro, Jal, Méx, Mich, Oax, Qro, Ver

**H. orizabensis F. A. Ley, Ver

*H. pachydiscus (Rydb.) Standl., CdMx, Gto, Hgo, Méx, Qro

Horkelia Cham. \& Schltdl.

Género de unas 20 especies del oeste de Norteamérica. Solamente dos en el extremo NW de Baja California. Referencia: Ertter \& Reveal, 2014.

H. clevelandii (Greene) Rydb., BC

H. truncata Rydb., BC

Ivesia Torr. \& A. Gray

Género de unas 30 especies del oeste de Norteamérica. Solamente dos en el NW de Baja California. Referencia: Ertter, 1989.

I. argyrocoma (Rydb.) Rydb., BC

I. saxosa (Greene) Ertter, BC

Lachemilla (Focke) Rydb.

Género de unas 80 especies americanas, distribuidas del suroeste de Estados Unidos de América a Argentina y Chile, mayormente habitantes de regiones con clima templado de montañas intertropicales. Con anterioridad considerado como una sección de Alchemilla.

Gehrke et al.(2008) reconocen el carácter monofilético de Lachemilla, pero con sus resultados sugieren reunir Alchemilla, Aphanes y Lachemilla en un solo género.

Referencias: Perry, 1929; Gehrke et al., 2008.

L. aphanoides (Mutis ex L.f.) Rothm., Ags, BCS, CdMx, Chih, Chis, Dgo, Gro, Gto, Hgo, Jal, Méx, Mich, Mor, Nay, Oax, Pue, Qro, SLP, Son, Tlax, Ver

**L. mexiquense Morales-Briones, Méx

**L. orizabensis Rydb., Ver

L. pectinata (Ruiz \& Pavón) Rothm., Chis, Hgo. Oax. Pue, Qro, Tlax, Ver

L. pinnata (Ruiz \& Pavón) Rothm., Chis, Méx, Pue

L. procumbens (Rose) Rydb., CdMx, Chih, Chis, Dgo, Gto, Hgo, Jal, Méx, Mich, Mor, NL, Oax, Pue, Sin, SLP, Tlax, Ver

L. sibbaldiifolia (Kunth) Rydb., CdMx, Chih, Chis, Dgo, Gto, Hgo, Jal, Méx, Mich, Mor, Oax, Pue, Qro, Sin, SLP, Tlax, Ver

*L. velutina (S. Watson) Rydb., Ags, CdMx, Gro, Gto, Hgo, Méx, Pue, Qro, SLP, Tlax, Ver

L. venusta (Schltdl. \& Cham.) Rydb., Chis, Oax, Pue

L. vulcanica (Schltdl. \& Cham.) Rydb., CdMx, Chis, Coah, Dgo, Jal, Méx., Mich, Oax, Pue, Qro, Tlax, Ver 
*Lindleya Kunth

Género mexicano de una sola especie calcífila, habitante de áreas con clima árido o semiárido. Referencia: Henrickson, 2012.

*Lindleya mespiloides Kunth, Ags, Chih, Coah, Dgo, Gto, Hgo, NL, Oax, Pue, SLP, Tamps, Ver, Zac

Malacomeles (Decne.) Decne.

Género esencialmente mexicano, extendiendo su área de distribución a Texas y Centroamérica; de sistemática controvertida, pues mientras algunos autores reconocen solamente dos especies, otros lo dividen en cinco. Aquí se reconocen tres. Referencias: Jones, 1945; Turner, 2011.

M. denticulata (Decne.) Decne., Ags, CdMx, Chis, Coah, Dgo, Gto, Hgo, Jal, Méx, NL, Oax, Pue, Qro, SLP, Tamps, Tlax, Ver, Zac

*M. paniculata (Rehder) J. B. Phipps, NL, Tamps

M. pringlei (Koehne) B. L. Turner, Chih, Coah, NL

Petrophytum (Nutt. ex Torr.) Rydb.

Género de tres especies, distribuido en el oeste y suroeste de Estados Unidos de América, así como en el norte de México, donde sólo se encuentra una. Referencia: Lis, 2014.

P. caespitosum (Nutt.) Rydb., Chih, Coah, NL, Tamps

Photinia Lindl.

Género de unas 60 especies, mayormente distribuidas en Asia. Unas pocas en México y Centroamérica. Referencia: Phipps, 1992.

**P. guerreris J. B. Phipps, Gro

P. matudae Lundell, Chis

*P. mexicana (Baill.) Hemsl., Chis, Mich, Oax, Ver

P. microcarpa Standl., Chis, Jal, Méx, Mich, Oax, Qro

*P. oblongifolia Standl., Jal, Nay

Physocarpus (Cambess.) Raf.

Género de unas 10 especies distribuido en Norteamérica y NE de Asia. Algunas se cultivan en calidad de ornamentales. Referencia: Alexander, 2014.

P. intemedius (Rydb.) C. K. Schneid., Coah, NL

Potentilla L.

Género de más de 500 especies de distribución casi cosmopolita pero particularmente diverso en regiones de clima templado del Hemisferio Norte. Su sistemática ha resultado excepcionalmente complicada, pues a menudo los límites entre los taxones son difíciles de definir, debido a la frecuente apomixis, hibridación e introgresión de caracteres.

Para México se diferencian alrededor de 35 componentes que requieren de revisión. Al parecer existe un contingente adicional por descubrir y describir.

Algunas especies antes incluidas en Potentilla han pasado a los géneros segregados Drymocallis, Horkelia e Ivesia. Varios autores prefieren reconocer también como independiente a Argentina Hill, representada en el país por P. anserina. A su vez, las plantas anteriormente ubicadas en Duchesnea están consideradas ahora como parte de Potentilla. Referencia: Rydberg, 1908.

P. anserina L., Chih, Mor

P. biennis DC., BC

**P. butandae Rzed. \& Calderón, Gto

*P. candicans Humb. \& Bonpl. ex Nestl., CdMx, Hgo, Méx, Mich, Mor, Pue, Tlax, Ver

**P. durangensis Rydb., Dgo

**P. ehrenbergiana Schltdl., Hgo

**P. exsul Standl., Chih

P. goldmanii Painter ex Rydb., Oax 
P. gracilis Hook., BC

*P. hamaetochrous Lehm., Gto, Hgo, Méx, Mich, Mor

$P$. heterosepala Fritsch, Chis, Gro, Méx, Pue, Ver

**P. horrida Rydb., Chih

${ }^{\circ}$ P. indica (Andrews) Th. Wolf, CdMx, Chis, Gro, Hgo, Méx, Mich, Oax, Pue, SLP, Tamps, Ver

**P. knoblochii Standl., Chih

*P. leonina Standl., Coah, NL

*P. leptopetala Lehm., Chih, Dgo

**P. luteosericea Rydb., BC

**P. macdonaldii B. L. Turner, Oax

**P. madrensis Rose, Nay

*P. mexiae Standl., Chih, Dgo

**P. oaxacana Rydb., Oax

P. oblanceolata Rydb., Chih

P. pensylvanica L., Tamps.

**P. queretarensis Rzed. \& Calderón, Qro

*P. ranunculoides Humb. \& Bonpl. ex Nestler, CdMx, Méx, Mich, Pue, Tlax, Ver

*P. richardii Lehm., Jal, Méx, Tlax, Ver

P. rimicola (Munz \& I. M. Johnst.) Ertter, BC

$P$. rivalis Nutt., $\mathrm{BC}, \mathrm{CdM}$, Mich

*P. rubra Willd. ex Schltdl., CdMx, Hgo, Méx, Mich, Ver

P. staminea Rydb., CdMx, Chis, Hgo, Jal, Méx, Mich, Tlax

P. subviscosa Greene, Son

P. supina L., Dgo, Zac

P. thurberi A. Gray, Chih, Coah, Dgo

**P. townsendii Rydb., Chih

\section{Prunus L.}

Género de unas 500 especies distribuidas mayormente en América y Eurasia. Incluye numerosos árboles frutales de relevante importancia económica. Entre los nativos de nuestro país cuenta el capulín (P. serotina ssp. capuli). Las especies mexicanas de Prunus requieren de una revisión crítica. Referencias: McVaugh, 1951; Pérez-Zabala, 2015; Standley, 1922.

$P$. axitliana Standl., Chis, Ver

*P. brachybotrya Zucc., Oax, Qro, Ver

**P. cercocarpifolia Villarreal, Coah

P. chiapensis Standl., Chis

*P. cortapico Kerber ex Koehne, Gro, Jal, Méx, Mich, Mor, Nay, Oax, Sin, Ver

P. emarginata (Douglas) Eaton, BC

*P. erythroxylon Koehne, Gro, Jal, Nay, Oax

$P$. fasciculata (Torr.) A. Gray, BC

P. fremontii S. Watson, BC

*P. gentryi Standl., Chih, Son

$P$. guatemalensis I. M. Johnst., Chis

P. havardii (W. Wight) S. C. Mason, Chih

**P. hintonii (C. K. Allen) Kosterm., Mich

P. ilicifolia (Nutt. ex Hook. \& Arn.) A. Dietrich, BC

P. lundelliana Standl., Chis

P. matudae Lundell, Chis

P. mexicana S. Watson, Coah, NL, SLP, Tamps

*P. microphylla (Kunth) Hemsl., Ags, CdMx, Dgo, Gto, Hgo, Méx, Oax, Pue, Qro, SLP, Tlax

P. myrtifolia (L.) Urb., Chis, Ver

**P. novoleontis Standl., NL

P. occidentalis Sw., Chis

${ }^{\circ}$ P. persica (L.) Batsch, BC

*P. prionophylla Standl., Gro, Jal, Méx, Mich, Oax 
P. rhamnoides Koehne, Chih, Chis, Dgo, Gto, Hgo, Jal, Mich, Oax, SLP, Tamps, Ver

P. salasii Standl., Chis

*P. samydoides Schltdl., Hgo, Oax, Pue, Qro, SLP, Ver

P. serotina Ehrh., Ags, BCS, CdMx, Chih, Chis, Coah, Col, Dgo, Gro, Gto, Hgo, Jal., Méx, Mich, Mor, Nay, NL, Oax, Pue, Qro, SLP, Sin, Son, Tamps, Tlax, Ver, Zac

*P. tartarea Lundell, Chis, Oax

*P. tetradenia Koehne, Chis, Hgo, Jal, Méx, Nay, Oax, Pue

*P. tuberculata Koehne, Chis, Oax, Qro

P. virginiana $\mathrm{L}$., $\mathrm{BC}$, Chih, Son

*P. zinggii Standl., Chih, Col, Gro, Jal, Mich, Mor, Nay, Oax, Son, Zac

\section{Purshia DC.}

Género de seis especies, distribuidas del suroeste de Canadá al centro de México. Referencia: McArthur et al., 1982.

P. ericifolia (Torr. \& A. Gray) Henrickson, Chih, Coah

*P. mexicana (D. Don) S. L. Welsh, Ags, Chih, Coah, Dgo, Gto, Jal, Son, Zac

*P. plicata (D. Don) Henrickson, Ags, Coah, Dgo, Gto, NL, SLP, Tamps, Zac

P. stanburyana (Torr.) Henrickson, BC, Son

P. tridentata (Pursh) DC., BC

${ }^{\circ}$ Pyracantha M. Roem.

Género de unas diez especies nativas de Eurasia. Varias se cultivan como ornamentales y algunas se han naturalizado en otros países.

${ }^{\circ} P$. angustifolia (Franch.) M. Roem., Chis

${ }^{\circ}$ P. crenulata M. Roem., Ver

${ }^{\circ}$ P. koidzumii (Hayata) Rehder, Mor, Pue

${ }^{o}$ Pyrus L.

Género de ca. 25 especies nativas de Eurasia y norte de África. Varias corresponden a árboles frutales ampliamente cultivados en el mundo entero.

${ }^{\circ} P$. communis L., BC

Rosa L.

Género de unas 150 especies distribuidas en Norteamérica, Eurasia y norte de África. Muchas se cultivan como ornamentales, medicinales y alimenticias y varias se han naturalizado en diferentes partes del mundo. Referencia: Lewis et al., 2014.

R. californica Cham. \& Schltdl., BC

${ }^{\circ}$ R. canina L., CdMx, Hgo, Méx, Mich, Mor, Ver

R. carolina L., Coah, NL, Tamps

R. minutifolia Engelm., BC

${ }^{\circ} R$. multiflora Thunb., Chis

$R$. woodsii Lindl., Coah

Rubus L.

Género de aproximadamente 700 especies de distribución casi cosmopolita. Al igual que Potentilla su sistemática ha resultado sumamente difícil de definir y abundan opiniones muy controvertidas acerca de la delimitación de sus elementos. El listado que a continuación se presenta está esencialmente basado en la contribución de Standley (1922).

Recientemente Alice et al. (2014) y Hernández Agudo (2018) han reducido a sinonimia a $R$. aboriginus, $R$. arizonensis, $R$. coriifolius, $R$. eriocarpus y $R$. schiedeanus. Aquí se conserva su vigencia. Además de las enlistadas existen en México aún especies por encontrar y describir. Los frutos de Rubus (frambuesas, zarzamoras) por lo general son comestibles y varias especies se cultivan para tal propósito, así como también en calidad de plantas ornamentales. Referencia: Standley (1922b). 
R. adenotrichos Schltdl., Chis, Col, Gro, Hgo, Jal, Méx, Mich, Mor, Oax, Pue, Ver

**R. alnifolius Rydb., Ver

${ }^{\circ} R$. armeniaca Focke, $\mathrm{BC}$

${ }^{\circ} R$. bifrons Vest, BC

R. coriifolius Liebm., Chis, Gro, Hgo, Méx, Mich, Oax, Ver

$R$. costaricanus Liebm., Chis

*R. cymosus Rydb., CdMx, Hgo, Jal, Méx, Mich, Qro

R. eriocarpus Liebm., CdMx, Chis, Gro, Méx, Oax, Pue, SLP, Tamps, Ver

R. fagifolius Schltdl. \& Cham., Chis, Ver

R. flagellaris Willd., Coah, Hgo, NL, SLP, Son

$R$. glaucus Benth., Chis, Oax

R. hadrocarpus Standl. \& Steyerm., Chis

$R$. humistratus Steud., Chis, Coah, Gto, Hgo, Jal, Mich, NL, Pue, Qro, SLP, Tamps, Ver

R. idaeus L., Chih

R. leptosepalus Donn.Sm., Chis

R. leucodermis Douglas ex Torr. \& A. Gray, Chih

R. liebmannii Focke, CdMx, Chis, Gro, Hgo, Méx, Mich, Mor, Oax, Ver

**R. macvaughianus Rzed. \& Calderón, Qro

R. miser Liebm., Chis

**R. nelsonii Rydb., Oax

R. neomexicanus A. Gray, Chih, Son

*R. palmeri Rydb., Chih, Dgo, Jal, Nay, Sin, Ver

R. parviflorus Nutt., Chih

*R. philyrophyllus Rydb., Hgo, Oax, Pue, Qro, Ver

R. pringlei Rydb., CdMx, Chih, Chis, Dgo, Gro, Gto, Jal, Méx, Mich, Mor, Oax, Pue, Ver

*R. pseudofagifolius Huan C. Wang, Oax, Ver

*R. pumilus Focke, CdMx, Chih, Dgo, Jal, Méx, Mich, Mor, Tlax

R. sapidus Schltdl., Chis, Hgo, Mich, Oax, Pue, Ver

R. schiedeanus Steud., Chis, Ver

$* * R$. sierrae J. E. Laferr., Chih

R. trilobus Ser., Chis, Gro, Oax, Pue, Tlax, Ver

R. trivialis Michx., Coah, NL, Tamps

*R. uhdeanus Focke, Méx, Mor

R. ursinus Cham. \& Schltdl., BC

R. urticifolius Poir., Chis, Oax

*R. verae-crucis Rydb., Hgo, Oax, Pue, Ver

\section{Sibbaldia L.}

Género recientemente reducido a solo cuatro especies distribuidas en áreas de clima templado y frío de Eurasia, Norteamérica, incluyendo el centro de México. Referencia: Eriksson et al., 2015.

S. procumbens L., Méx, Pue, Ver

Vauquelinia Correa ex Humb. \& Bonpl.

Género esencialmente mexicano de taxonomía complicada y controvertida, también presente en el SW de Estados Unidos de América; sus tres especies aceptadas son habitantes de áreas con clima árido. Referencia: Hess \& Henrickson, 1987.

*V. australis Standl., Oax, Pue

V. californica (Torr.) Sarg., BC, BCS, Chih, Coah, Dgo, Son

V. corymbosa Humb. \& Bonpl., Chih, Coah, Gto, Hgo, NL, Qro, SLP, Tamps

*Xerospiraea Henrickson

Género monotípico, endémico de ambientes áridos del norte y este de México. Referencia: Henrickson, 1985.

*X. hartwegiana (Rydb.) Henrickson, Coah, Dgo, Hgo, Oax, Pue, Qro, Ver. 


\section{LITERATURA CITADA}

Alexander, C. (2014). Physocarpus, En Fl. N. Amer. 9 (consultado en línea). www.efloras.ofg/florataxon.aspx?flora_id=18taxon_id=125317

Alice, L. A., Goldman, D. H., Macklin, J. A. \& Moore, G. (2014). Rubus. En Fl. N. Amer. 9: $28-56$.

Arce, G. L. \& Marroquín, J. S. (1985). Las unidades fisonómico florísticas del cañón de San Lorenzo, Coah., México. Biotica, 10, 369-393.

Barrie, F. R., Davidse, G. \& Pérez-Zavala, J. A. (2015). Rosaceae. En Flora Mesoamericana 2(3): 1-110.

Bitter, F. A. G. (1910). Die Gattung Acaena. Biblioth. Bot., 17(74), 1-336.

Calderón de Rzedowski, G. (2001). Rosaceae. En Flora fanerogámica del Valle de México. (págs. 233-250). Pátzcuaro, Mich. Instituto de Ecología, A. C., Centro Regional del Bajío.

Campbell, C. S. (2014). Malacomeles. En Fl. N. Amer. 9 (consultado en línea). www. efloras.org/florataxon.aspx?flora_id $=18$.taxon_id $=119515$

Colecciones Biológicas UNAM. datosabiertos.unam.mx

Encina Domínguez, J. A., Gómez Pérez, S. G. \& Valdés Reyna, J. (2012). Composición florística y ecología del matorral de rosáceas de la sierra de Zapalinamé, Coahuila, México. J. Bot. Res. Inst. Texas, 6, 143-156.

Eriksson, T., Lundberg, M., Töpel, M., Östensen, P. \& Smedmork, J. E. E. (2015). Sibbaldia, a molecular study of a remarkably polyphyletic genus of Rosaceae. Pl. Syst. Evol., 301, 171-184.

Ertter B. (1989). Revisionary studies in Ivesia (Rosaceae). Syst. Bot., 14, 231-244.

Ertter, B. (2007). Generic realignment in the tribe Potentilleae and revision of Drymocallis. J. Bot. Res. Inst. Texas, 1, 31-46.

Ertter, B. \& J. L. Reveal. (2014). Horkelia. En Fl. N. Amer 9 (consultado en línea).

Gajewski, W. (1956). A cytogenetic study of the genus Geum L. Monogr. Bot., 4, 1-416.

GBIF, www.gbif.org

Gehrke, B., Bräucher, C., Romoleroux, K., Lundberg. G. Heubl, M., \& Ericksson, T. (2008). Molecular phylogenetics of Alchemilla, Aphanes and Lachemilla (Rosaceae) inferred from plastid and nuclear intron and spaces DNA sequences, with comments on generic classification. Molec. Phylogen. Evol., 47, 1030-1044.

Henrickson, J. (1985). Xerospiraea, a generic segregate of Spiraea (Rosaceae). Aliso, 11, 199-211.

Henrickson, J. (2001). Systematics and relationships of Fallugia (Rosoideae - Rosaceae). Aliso, 20, 1-15. 
Henrickson, J. (2012). Systematics of Lindleya (Rosaceae: Maloideae). J. Bot. Res. Inst. Texas, 6, 341-360.

Henrickson, J. (2014). Chamaebatia. En: Fl. N. Amer 9 (consultado en línea). www.efloras.org/florataxon.aspx?flora_id=18taxon_id=106415

Henrickson, J. \& Johnston, M. C. (1997). Rosaceae. En. A flora of the Chihuahuan Desert region. (pp. 606-629). Los Angeles, California. Published by James Henrickson.

Hernández Agudo, F. O. (2018). Revisión taxonómica del género Rubus (Rosaceae) en Costa Rica y Panamá. Tesis. (130 págs.). San José. Universidad de Costa Rica.

Hess, W. L. \& J. Henrickson. (1987). A taxonomic revision of Vauquelinia. Sida, 12, 101-163.

Jones, G. N. (1945). Malacomeles, a genus of Mexico and Guatemala. Madroño, 8, 33-39.

Kline, G. J. \& Sorensen, P. D. (2008). A revision of Agrimonia in North and Central America. Brittonia, 60, 11-33.

Lewis, W. H., B. Ertter \& Bruneau, A. (2014). Rosa. En Fl. N. Amer. 9 (consultado en línea). www.efloras.org/florataxon.aspx?flora_id=18taxon_id=128746

Ley, A. (1943). A taxonomic revision of the genus Holodiscus (Rosaceae). Bull. Torr. Bot. Club, 70, 275-288.

Lis, R. A. (1990). A taxonomic revision of Holodiscus Maxim. (Spiraeoideae: Rosaceae) based upon numerical analyses of morphometric variation. Ph.D. dissertation. (414 págs.). Berkeley. University of California.

Lis, R. A. (1992). Leaf architecture survey of Cercocarpus (Rosaceae) and its systematic significance. Intern. J. Pl. Sci., 153, 258-272.

Lis, R. A. (2014). Petrophytum. En Fl. N. Amer. 9 (consultado en línea). www.efloras.org/florataxon.aspx?flora_id $=18$ taxon_id $=31468$

Martin, F. L. (1950). A revision of Cercocarpus. Brittonia, 7, 91-111.

Martínez-Ramírez J. (2014). Rosaceae, En Flora del Valle de Tehuacán-Cuicatlán 120, 1-62.

McArthur, E. R., Stutz, H. C. \& Sonderson, S. C. (1982). Taxonomy, distribution and cytogenetics of Purshia, Cowania and Fallugia (Rosoideae, Rosaeae). (págs. 4-31). Ogden, Utah. U. S. Department of Agriculture. Forest Service Rep. INT-182.

McNeill, J. \& B. Ertter. (2014). Aphanes. En Fl. N. Amer. 9 (consultado en línea).

McVaugh, R. (1951). A revision of the North American black cherries (Prunus serotina and relatives). Brittonia, 7, 279-315.

Pérez-Zabala, J. A. (2015). Prunus. En Flora Mesoamericana 2(3), 31-47.

Perry, L. M. (1929). A tentative revision of Alchemilla section Lachemilla. Contr. Gray Herb., 84, 3-57.

Phipps, J. B. (1997). Monograph of northern Mexican Crataegus (Rosaceae, subfam. Maloideae). Sida Bot. Misc., 15, 1-94. 


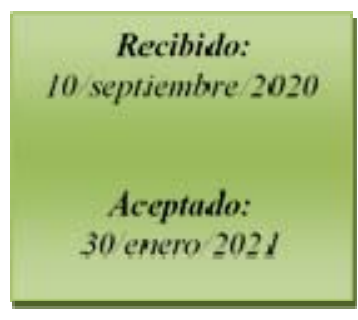

Phipps, J. B. (1992). Heteromeles and Photinia (Rosaceae, subfam. Maloideae) of Mexico and Central America. Canad. J. Bot., 70, 2138-2162.

Potter, D., Gao, F., Bortin, P. E., Oh, S. \& Beggett, S. (2002). Phylogenetic relationships in Rosaceae inferred from chloroplast matK and trnL-trnF nucleotide sequence data. Pl. Syst. Evol., 231, 77-89.

Rebman, J. P., Gibson, J. \& Rich, K. (2016). Rosaceae. En Annotated checklist of the vascular plants of Baja California, Mexico. Proc. San Diego Soc. Natur. Hist., 45, 244-247.

Rydberg, P. A. (1908-1918). Rosaceae. En N. Amer. Fl., 22, 239-533.

Rydberg, P. A. (1908). Potentilla. En N. Amer. Fl., 22, 293-388.

Rzedowski, J. \& Calderón de Rzedowski, G. (2006). Rosaceae. En Flora del Bajío y de regiones adyacentes, 13, 1-157.

Spellenbeerg, R., Lebgue, T., Corral-Díaz, R. \& Bacon, S. (1995). Nuevos registros de plantas de las montañas del norte de México. Acta Bot. Mex., 30, 13-20.

Standley, P. C. (1922). Rosaceae, Malaceae \& Amygdalaeae. En Trees and shrubs of Mexico. Contr. U.S. Natl. Herb., 23, 321-345.

Standley, P. C. (1922a). Adenostoma. En Trees and shrubs of México. Contr. U.S. Natl. Herb., 23, 325.

Standley, P. C. (1922b). Prunus. En Trees and shrubs of Mexico. Contr. U.S. Natl. Herb., 23, 338-343.

Standley, P. C. (1922c). Rubus. En Trees and shrubs of Mexico. Contr. U.S. Natl. Herb., $23,328-333$.

Staudt, G. (1999). Systematics and geographic distribution of the American strawberries. Taxonomic studies in the genus Fragaria (Rosaceae: Potentilleae). Univ. Calif. Publ. Bot.. $81,1-162$.

Tropicos, Missouri Botanical Garden, www.tropicos.org

Turner, B. L. (2011). Recension of the genus Malacomeles (Rosaceae), Phytologia, 93, 99106.

Varios autores. (2014). Rosaceae. En Fl. North Amer. 9 (consultado en línea). www.efloras.org/florataxon.aspx?flora_id=18taxon_id=10976

Vásquez-Cruz, M. \& Sosa, V. (2020). Assembly and origin of the flora of the Chihuahuan Desert: the case of sclerophyllous Rosaceae. J. Biogeogr., 47, 445-459.

Velasco de León, P. \& Cevallos-Ferriz, S. R. S. (2000). Leaves of Cercocarpus mixteca n. sp. from Oligocene sediments, near Tepexi de Rodríguez, Puebla. Rev. Paleont. Palynol., $111,285-294$.

Villaseñor, J. L. (2016). Rosaceae. En Checklist of the native vascular plants of Mexico. Rev. Mex. Biodivers., 87, 868-870. 